\title{
The influence of antibiotic-loaded cement spacers on the risk of reinfection after septic two-stage hip revision surgery
}

\author{
Kevin Staats ${ }^{1} \cdot$ Florian Sevelda $^{1} \cdot$ Alexandra Kaider $^{2} \cdot$ Christoph Böhler $^{1}$. \\ Irene K. Sigmund ${ }^{1} \cdot$ Stephan E. Puchner $^{1} \cdot$ Reinhard Windhager $^{1} \cdot$ Johannes Holinka $^{1}$
}

Received: 26 March 2017 / Accepted: 11 October 2017 / Published online: 24 October 2017

(c) The Author(s) 2017. This article is an open access publication

\begin{abstract}
Purpose The aim of this study was the evaluation of possible outcome differences of patients undergoing two-stage hip exchange with antibiotic-loaded spacers, compared to patients without an interim spacer implantation.

Methods We evaluated 46 patients undergoing two-stage hip revision surgery. Twenty-five patients received an interim ALS. Additional to a Kaplan-Meier survival analysis, a competing risk analysis was performed to estimate the cumulative incidence function for re-revisions due to infection accounting for death as a competing event.

Results Nine patients (seven non-ALS vs. two ALS) had to undergo re-revision surgery due to reinfection of the hip joint. The non-ALS group showed a risk of re-revision of $19 \%$ (95\% CI 5-38\%) at 12 and 24 months and 30\% (95\% CI $12-51 \%)$ at 36 months. The group with ALS implantation displayed a $0 \%$ risk of re-revision surgery in the first 36 months. The Gray test revealed a significant difference in the cumulative incidence between both observed groups $(p=0.026)$.

Conclusion Our findings suggest that ALS implantation significantly reduces the risk of reinfection after two-stage hip revision surgery.
\end{abstract}

Keywords Periprosthetic joint infection $\cdot$ Hip $\cdot$ Spacer

Kevin Staats

kevin.staats@meduniwien.ac.at

1 Department of Orthopaedic Surgery, Medical University of Vienna, Waehringer Guertel 18-20, 1090 Vienna, Austria

2 Section for Clinical Biometrics, Centre for Medical Statistics, Informatics, and Intelligent Systems, Medical University of Vienna, Waehringer Guertel 18-20, 1090 Vienna, Austria

\section{Introduction}

Periprosthetic joint infection (PJI) is one of the most demanding complications after total hip arthroplasty (THA), it has a vast impact on patient's morbidity and mortality, and remains a socio-economic problem [1-4]. The best therapeutic strategy for PJI still remains open to widespread debate $[1,5-9]$. Two-stage revisions show satisfying success rates, at around $90 \%$, and therefore, represent the standard procedure especially for late/chronic infections $[6,9,10]$. During the first-stage procedure, the infected prosthesis is removed and an implantation of an interim antibiotic-loaded cement spacer (ALS) can be considered. The major advantage of the usage of ALS is the possibility of maintaining high antibiotic concentrations that reach a local therapeutic level [11]. Therefore, ALS may contribute to the eradication of a PJI, as ALS improves the antimicrobial efficacy of systemic antibiotics [12]. An additional benefit may be the avoidance of possible contractures through the reduction of the dead space after explantation of the infected implants $[6,13]$.

Despite the broad usage of ALS in two-stage revisions, there still remains concern in regard to induction of resistances and development of biofilm-forming microorganisms [14-17]. In the worst-case scenario, the spacer itself becomes-due to its surface conditions-a vehicle for adherent microorganisms [12, 18]. Zimmerli et al. claim that only in cases where no "difficult-to-treat"(DTT)-microorganisms (MRSA, small colony variants of staphylococci, enterococci, quinolone-resistant Pseudomonas aeruginosa and fungi) are isolated, should an ALS be implanted during the first-stage procedure [19]. Additionally, with the use of rather new diagnostic tools, spacers seem to be more colonized by microorganisms than priorly expected [20,21].

$\mathrm{We}$, therefore, raised the question of whether the treatment of ALS has an effect on the re-revision rate and risk of 
reinfection in patients undergoing a two-stage procedure due to PJI in THA. We hypothesized that ALS has a benefit on the outcome parameters and cause a reduction in the reinfection rate, regardless of the type of microorganism.

\section{Patients and methods}

We performed a retrospective study of patients suffering from a deep infection after total hip arthroplasty (THA) between 2001 and 2014 using the hospital database. The local ethics committee approved the study.

PJI was suspected preoperatively by the presence of leucocytosis, elevated C-reactive protein-levels (CRP), pain, swelling, local erythema and warmth. PJI was verified intraoperatively through positive microbiology and/or positive histopathological examination. Upon examining positive culture results, so-called "difficult-to-treat" microorganisms were registered. These DTT microorganisms involved Methicillin-resistant Staphylococcus aureus (MRSA), small colony variants of staphylococci, enterococci, quinolone resistant $P$. aeruginosa and fungi. [19, 22].

46 patients ( 21 female and 25 male, mean age $=64$ years, range $24-87$ years) were included in this study. Twenty-one patients underwent two-stage revision without an interim ALS (example Fig. 1) and 25 patients received an ALS after the first stage (example Fig. 2). No randomization was performed, regardless of whether patients were provided with an ALS or without ALS implantation. ALS usage was dependent solely upon the surgeon's choice.

For all ALSs included, the same antibiotic bone cement, containing $0.5 \mathrm{~g}$ of Gentamicin and $2 \mathrm{~g}$ of Vancomycin per $40 \mathrm{~g}$ bone cement $\left(\mathrm{COPAL}^{\circledR} \mathrm{G}+\mathrm{V} 40\right.$, Heraeus, Wehrheim, Germany) was used. In the ALS group a custom-moulded articulating spacer system (Stage One ${ }^{\circledR}$, Biomet, Warsaw, USA) was implanted. All patients received intravenously administered antibiotics 30 min prior to surgery and for at least 4 weeks postoperatively with 10-14 days iv-administration. If a pathogen was detected, the treatment was then assessed following performance of an antibiogram and a resistogram and adjusted accordingly if required. Treatment

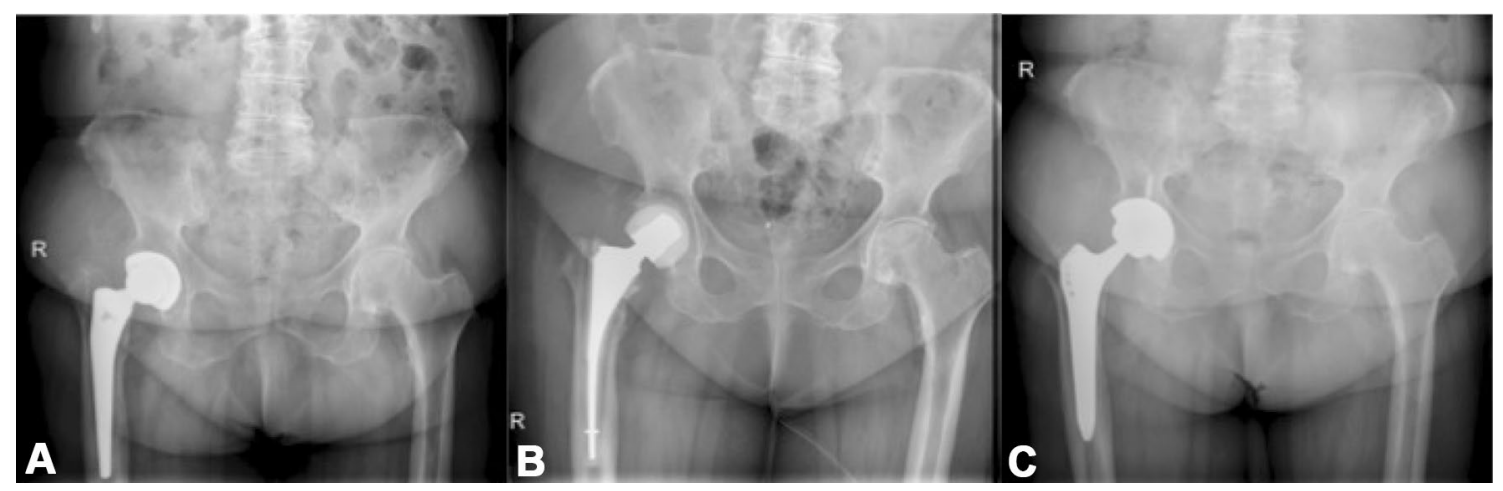

Fig. 1 Example of a female patient with hip arthroplasty of the right hip joint (a) who had undergone two-stage revision surgery due to periprosthetic joint infection. Interim antibiotic-loaded spacer was implanted between explantation (b) and reimplantation (c)

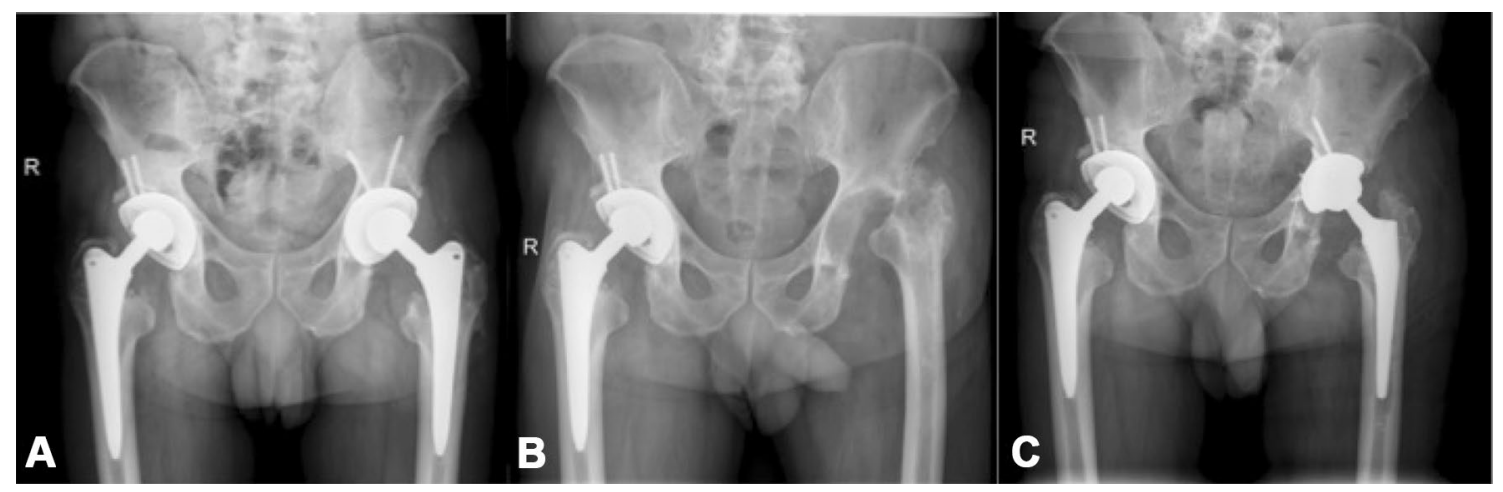

Fig. 2 Example of a male patient with bilateral hip arthroplasty (a) who had undergone two-stage revision surgery due to periprosthetic joint infection on the left side. No interim antibiotic-loaded spacer was implanted between explantation (b) and reimplantation (c) 
adjustments were performed using the antibiotic treatment recommendations by Trampuz and Zimmerli [19]. If patients were discharged before reimplantation, the antibiotics were switched to orally administered options in accordance with prior consultation with our specialist for infectious diseases. Reimplantation was planned following a minimum of 4 weeks from the date of explantation. During this interstage period, CRP and leucocyte levels were examined on a regular basis. After reimplantation, all patients received iv-antibiotics for at least 7 days, which were then changed to oral antibiotics. These antibiotics were then administered for a period of time ranging from 21 days up to 5 weeks.

Since patient-specific comorbidities predispose for periprosthetic infections [23], systemic host factors and infection types were evaluated using the scoring system described by McPherson et al. [8]. Patients with no compromising host factors were classified as systemic host grade A, patients with one or two compromising factors were assigned to systemic host grade B and patients with three or more compromising factors were classified as systemic host grade $\mathrm{C}$. Table 1 exemplifies those compromising host factors. This classification system also distinguishes between early infections occurring in the first 4 weeks after primary implantation, haematogenous infection also occurring in the first 4 weeks but usually with previous well-functioning joint and presence of bacteremia and late (chronic) infection with symptoms occuring after 4 weeks after primary implantation. Table 2 displays the detected pathogens and inflammatory parameters during initial infection for both groups.

\section{Statistical analysis}

To evaluate the differences in possible confounding parameters between the study group and the control group, the Mann-Whitney $U$ test (for numerical and ordinal variables) and the Chi-square test (for binary variables) were applied. Revision-free survival and cumulative survival was calculated using a Kaplan-Meier survival analysis. A log-rank test was applied to detect differences between the observed groups.

Since patients undergoing hip revision arthroplasty show a significant increase in postoperative mortality [24, 25], competing risk analysis was performed to estimate the cumulative incidence function for re-revisions, due to infection accounting for death as a competing event. The cumulative probabilities are given together with the $95 \%$ confidence intervals (95\% CI). Gray's test was used to test for statistically significant differences between observed group and $p$ values of $<0.05$ were considered as statistically significant. Statistical analysis was performed using SPSS software, version 23.0 (SPSS Inc., Chicago, USA) and SAS, version 9.4 (SAS Institute Inc., Cary, NC, USA).

\section{Results}

From a total of 46 included patients who received two-stage hip revision surgery, 9 patients (7 non-ALS vs. 2 ALS) had to undergo re-revision surgery due to reinfection of the hip joint. No differences were found in basic demographics between the two groups (Table 3). The mean followup was 46 months (range 12-139 months). Kaplan-Meier analysis revealed a revision-free survival in the non-ALS
Table 1 Compromising host factors following the grading system by McPherson et al. [8]

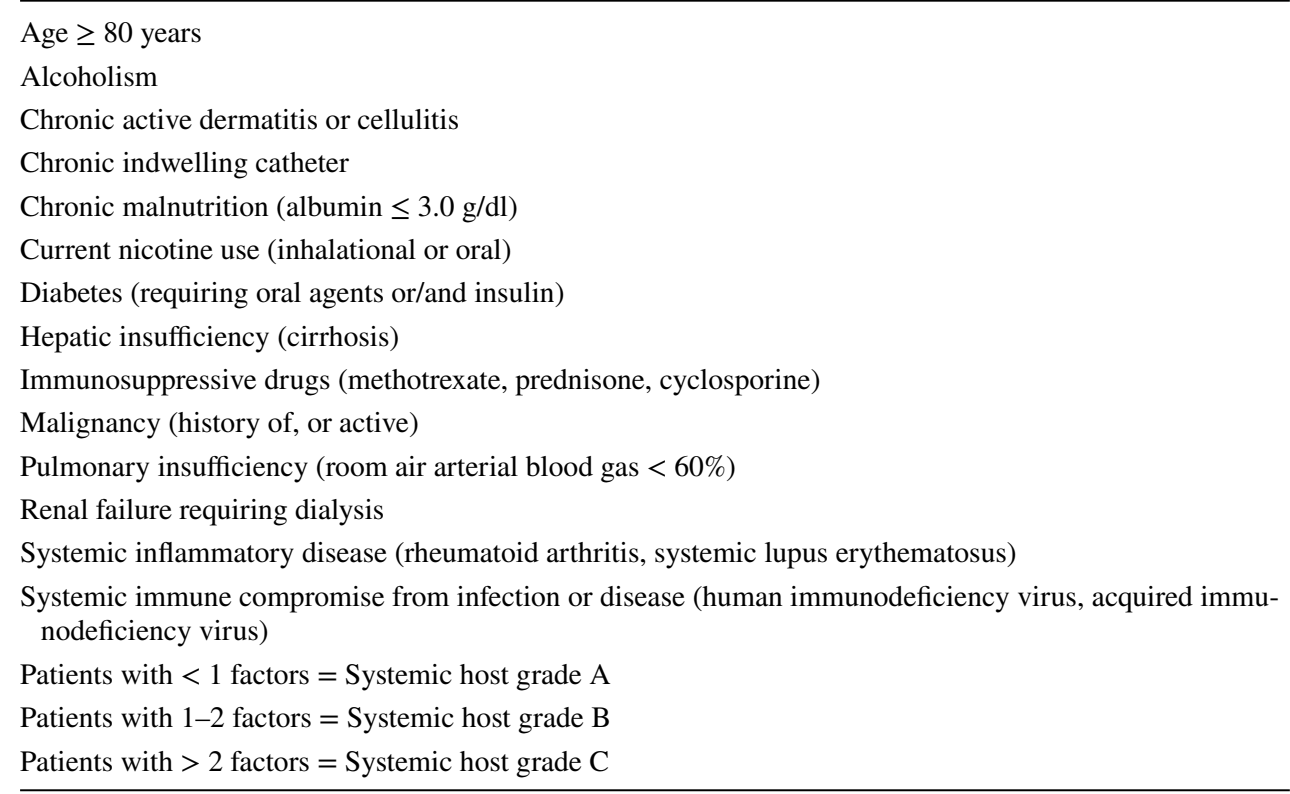


Table 2 Pathogens and immediate preoperative inflammatory parameters during initial infection

\begin{tabular}{|c|c|c|}
\hline & No ALS implantation $(n=21)$ & ALS implantation $(n=25)$ \\
\hline Pathogen detected at initial infection & $\begin{array}{l}\text { Corynebacterium spp.: } n=2 \\
\text { Enterobacter cloacae: } n=1 \\
\text { Enterococcus faecium: } n=1 \\
\text { Propionibacterium acnes: } n=1 \\
\text { Pseudomonas aeruginosa: } n=1 \\
\text { Staph. } \text { epidermidis: } n=2 \\
\text { Staph. } \text { aureus: } n=1 \\
\text { MRSA: } n=1 \\
\text { No pathogen detected: } n=9\end{array}$ & $\begin{array}{l}\text { Corynebacterium spp.: } n=1 \\
\text { Enterobacter cloacae: } n=1 \\
\text { Enterococcus faecium: } n=1 \\
\text { Escherichia coli: } n=1 \\
\text { Propionibacterium acnes: } n=1 \\
\text { Staph. caprae: } n=1 \\
\text { Staph. } \text { epidermidis: } n=3 \\
\text { Staph. aureus: } n=1 \\
\text { MRSA: } 7 \\
\text { Beta-haemolytic Streptococci: } n=1 \\
\text { Viridans streptococci: } n=1 \\
\text { No pathogen detected: } n=7\end{array}$ \\
\hline CRP preoperative (explantation) & $\begin{array}{l}\text { Mean: } 6.1 \mathrm{mg} / \mathrm{dL} \\
\text { (range: } 0.72-41.69 \mathrm{mg} / \mathrm{dL} \text { ) }\end{array}$ & $\begin{array}{l}\text { Mean: } 9,25 \mathrm{mg} / \mathrm{dL} \\
\text { (range: } 1,06-41,93 \mathrm{mg} / \mathrm{dL} \text { ) }\end{array}$ \\
\hline Leukocyte count & $\begin{array}{l}\text { Mean: } 12.54 \mathrm{~g} / \mathrm{L} \\
\text { (range: } 8.42-21.83 \mathrm{~g} / \mathrm{L} \text { ) }\end{array}$ & $\begin{array}{l}\text { Mean: } 11.56 \mathrm{~g} / \mathrm{L} \\
\text { (range: } 7.09-19.55 \mathrm{~g} / \mathrm{L} \text { ) }\end{array}$ \\
\hline Histopathological results & $\begin{array}{l}\text { Positive for infection: } n=18 \\
\text { Negative for infection: } n=3\end{array}$ & $\begin{array}{l}\text { Positive for infection: } n=22 \\
\text { Negative for infection: } n=3\end{array}$ \\
\hline
\end{tabular}

In one patient of the non-ALS group, the same pathogen (Staphylococcus epidermidis) could be detected during re-revision surgery In all remaining patients (non-ALS and ALS) with additional surgery, no pathogen could be detected

Table 3 Demographics of patients who received an implantation of an interim antibiotic-loaded cement spacer (ALS) compared to patients without ALS implantation

\begin{tabular}{llll}
\hline & No ALS implantation $(n=21)$ & ALS implantation $(n=25)$ & $p$ value \\
\hline Age (mean) & $61( \pm 15.5)$ & $67( \pm 12.1)$ & 0.15 \\
Gender & Female: $52.4 \%(n=11)$ & Female: $40 \%(n=10)$ & 0.553 \\
& Male: $47.6 \%(n=10)$ & Male: $60 \%(n=15)$ & \\
Systemic host grade [8] & A: $28.6 \%(n=6)$ & A: $36 \%(n=9)$ & 0.424 \\
& B: $61.9 \%(n=13)$ & B: $44 \%(n=11)$ & \\
& C: $9.5 \%(n=2)$ & C: $20 \%(n=5)$ & 0.585 \\
Infection type [8] & Early $(<4$ wks): $9.5 \%(n=2)$ & Early $(<4$ weeks $): 4 \%(n=1)$ & \\
& Hematogenous $(<4$ wks): $0 \%$ & Hematogenous $(<4$ weeks $): 0 \%$ & \\
& Late $(\geq 4$ wks): $90.5 \%(n=19)$ & Late $(\geq 4$ weeks): $96 \%(n=24)$ &
\end{tabular}

Systemic host grade A represents patients without any compromising host factors (comorbidities), grade B involves patients with 1-2 compromising factors and grade $\mathrm{C}$ equals $>2$ compromising factors

Compromising Host Factors are listed in Table 1 group of $76.1 \%(n=16)$ at 12 months, $71.4 \%(n=15)$ at 24 months and a long-term revision-free survival rate of $66.6 \%(n=14)$. The group with ALS implantation showed a revision-free survival rate of $100 \%(n=25)$ at 12 months, $100 \%(n=25)$ at 24 months and a long-term revision-free survival rate of $92 \%(n=23)$. Log-rank test revealed a significantly better revision-free survival in the ALS group compared to the non-ALS group ( $p=0.036$ ) (Fig. 3).

After reimplantation, one patient of the ALS died 16 months after reimplantation. In the non-ALS group, two patients died 15 and 91 months, respectively, after reimplantation. Therefore, for the ALS cohort, an estimation of cumulative incidences revealed an overall risk of re-revision surgery after the second stage of $8.7 \%$ (95\% CI 3-19\%) at 12 (42 patients followed-up) and 24 months (33 patients followed-up) and $14.6 \%$ (95\% CI 6-28\%) at 36 months (24 patients followed-up). The non-ALS group showed a risk of re-revision of $19 \%$ (95\% CI 5-38\%) at 12 and 24 months and $30 \%$ (95\% CI 12-51\%) at 36 months. The group with ALS implantation displayed a risk of re-revision surgery of $0 \%$ in the first 36 months. At 60 months (nine patients followed-up), the risk of re-revision increased to $10 \%$ in 


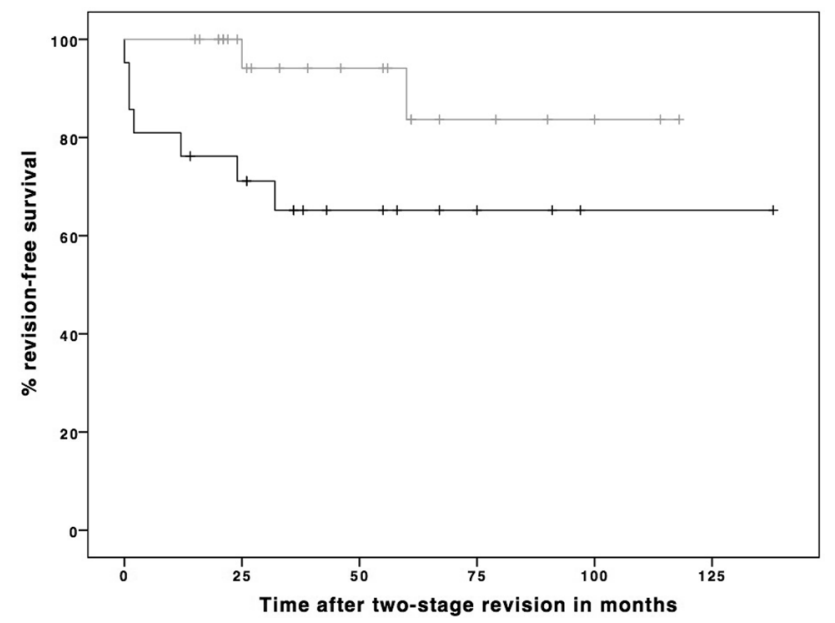

Fig. 3 Kaplan-Meier survival curve showing significant $(p=0.036)$ better revision-free survival in patients with interim antibiotic-loaded spacer (gray) compared to patients without an interim antibioticloaded spacer (black)

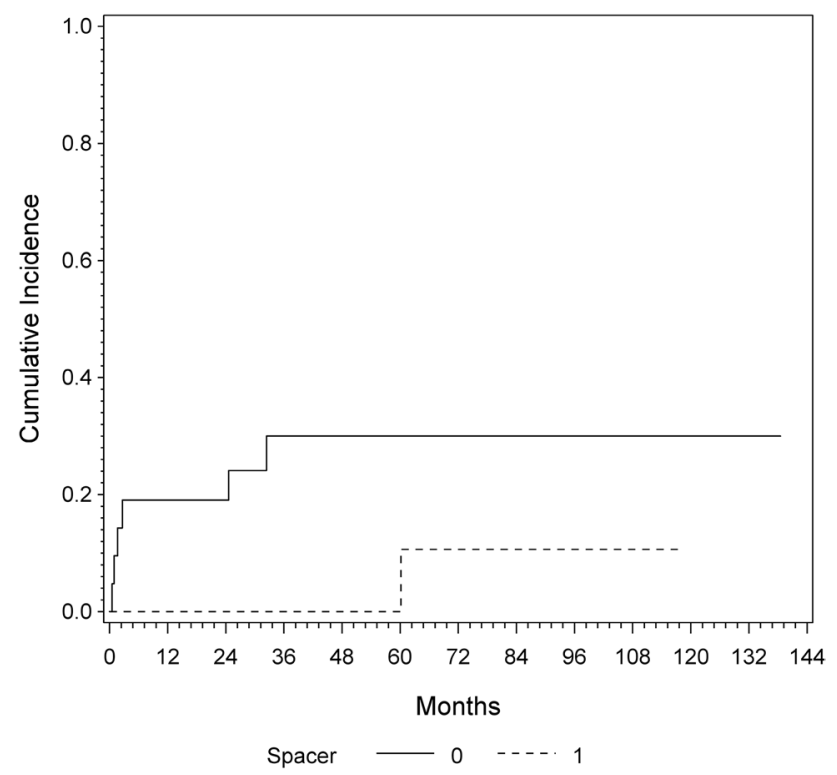

Fig. 4 Cumulative incidence of re-revision in patients with implantation of antibiotic-loaded spacer (dotted) and without interim antibiotic spacer (line), assessed by competing risk analysis

this group. The Gray test revealed a significant difference in the cumulative incidence between both observed groups $(p=0.026)$ (Fig. 4).

In regard to the aforementioned "difficult-to-treat" (DTT) microorganisms, seven patients in the ALS group were initially infected with DTT of whom six patients suffered from an infection with Methicillin-resistant Staphy. aureus (MRSA), and in one patient MRSA and Enterococcus faecalis was found. One of those seven patients had to undergo additional revision surgery and further antibiotic treatment due to reinfection. Reduced susceptibility to Vancomycin could not be detected in this case. In the group without ALS implantation, one patient suffered from MRSA and another patient from E. faecalis infection. Both patients had to undergo re-revision surgery, but no pathogen could be detected during these additional revision surgeries. Only in one patient of the non-ALS group, the same pathogen (Staphy. epidermidis) could be detected during re-revision surgery. In all remaining patients (both ALS and non-ALS) with additional surgery, no pathogen could be detected.

\section{Discussion}

Two-stage revision surgeries represent a standard procedure for the treatment of periprosthetic joint infections (PJI). Antibiotic-loaded cement spacers (ALS) are broadly used as an interim replacement for the interval between explantation and reimplantation. ALS implantation not only leads to higher local antibiotic-levels between first and second stage surgery [26], it also improves the functional and satisfactory outcome in terms of an improved joint mobility and a lower contracture rate [27]. ALSs also facilitate reimplantation due to minimizing leg length discrepancy [28, 29]. Nevertheless, concern still exists whether ALS should be implanted in two-stage revisions by default $[9,19,30,31]$.

We, therefore, raised the question of if ALS implantation reduces the risk of reinfection after two-stage revisions due to periprosthetic hip infection.

After thorough research on this topic, this is the first single-center study to compare two-stage hip revisions with or without interim ALS implantation in regard to the risk of reinfection.

The fact that the use of an interim ALS declined the rerevision rate and improved the outcome with regards to a reduced risk of reinfection represent major findings in this study. From our perspective, the use of ALS in two-stage hip revisions is recommended. These recommendations are supported by the fact that besides establishing the use of ALS in two-stage hip revision cases in our department in 2008 , no further changes regarding surgical approach and systemic antibiotic treatment have been perceived during the whole observational period between both groups in this retrospective cohort study. Therefore, it seems conceivable that the decline in the re-revision rate and reduced risk of reinfection is due to the use of ALS.

In the literature, two-stage revisions deliver the highest success rate with re-revision rates of about 10\% [32]. Despite a high overall re-revision rate of $19.6 \%$, ALS implantation decreased revisions after two-stage exchange and lead to a long-term implant survival rate of $92 \%$. These results confirm the findings of Chen et al., where they gathered similar reinfection rates in two-stage revisions with ALS [33]. 
In spite of this, ALS represents a foreign body that is implanted in the infected joint. This may lead to a slowdown, or even an inhibition of PJI eradication [34]. Therefore, some authors discourage the non-restrictive use of ALS [22, 34, 35].

In 2015, Gomez et al. investigated the outcome of twostage revisions in the treatment of periprosthetic infection [15]. They found that a substantial amount of patients do not undergo reimplantation after spacer implantation and about one-fifth of patients needed revision after reimplantation. In our cohort, only one patient underwent spacer exchange due to persistent infection and the overall re-revision rate corresponds to the results by Gomez et al. However, static and articulating spacers were included in their study, whereas only articulating spacers were used in our investigation, and articulating spacers seem to produce better results than static spacers [36, 37].

Zimmerli et al. state that surgeons should refrain from using ALS implantation in cases with "difficult-to-treat" (DTT) microorganisms. Reason for this restriction is that the ALS itself may represent a pathogen vehicle, especially in cases caused by antibiotic-resistant pathogens. The ALS may then eventually contribute to biofilm formation and the treatment may become more complex and difficult. These DTT microorganisms involve Methicillin-resistant Staphy. aureus (MRSA), small colony variants of staphylococci, enterococci, quinolone resistant $P$. aeruginosa and fungi. $[19,22]$. Due to the low number of DTT cases in this study, we cannot give distinct evidence to discourage the reader from this recommendation. Nonetheless, we still found a tendency for a better outcome for DTT infections with ALS implantation. However, studies with larger sample sizes are needed to confirm this statement.

This study shows some limitations: First, due to its retrospective character and the long observation period, some selection bias can be expected. This selection bias may be due to the fact that 17 out of the 21 patients who did not receive ALS were treated between 2001 and 2008, and 21 out of 25 patients with ALS implantation were treated after 2008. Therefore, the better outcome of the ALS group may also be due to the fact that surgical techniques and antibiotic treatment improved during the observation period. Second, the relatively low number of patients may lead to an over- or underestimation of the results, but our sample size seems similar with other studies dealing with this subject, and therefore, our results remain comparable.

\section{Conclusion}

Our findings suggest that ALS implantation reduces the risk of reinfection after two-stage hip revision surgery. These results support the position that $A B$ spacers fulfil the requirements of effective $\mathrm{AB}$ levels and seem to achieve a broad bacterial coverage. However, larger studies are needed to confirm this statement for infection with pathogens, which are known as difficult to treat. Additionally, since the development of $\mathrm{AB}$ resistances and the occurrence of biofilmforming microorganisms increase, further investigations regarding new developments in diagnostics and treatment strategies should be obtained constantly.

Acknowledgements Open access funding provided by Medical University of Vienna.

\section{Compliance with ethical standards}

Conflict of interest On behalf of all authors, the corresponding author states that there is no conflict of interest.

Funding There is no funding source.

Ethical approval The study was approved by the local ethics committee.

Informed consent Informed consent was obtained from all individual participants included in the study.

Open Access This article is distributed under the terms of the Creative Commons Attribution 4.0 International License (http://creativecommons.org/licenses/by/4.0/), which permits unrestricted use, distribution, and reproduction in any medium, provided you give appropriate credit to the original author(s) and the source, provide a link to the Creative Commons license, and indicate if changes were made.

\section{References}

1. Berend KR, Lombardi AV, Morris MJ, Bergeson AG, Adams JB, Sneller MA. Two-stage treatment of hip periprosthetic joint infection is associated with a high rate of infection control but high mortality. Clin Orthop Relat Res. 2013;471:510-8.

2. Bedair H, Goyal N, Dietz MJ, Urish K, Hansen V, Manrique J, et al. A history of treated periprosthetic joint infection increases the risk of subsequent different site infection. Clin Orthop Relat Res. 2015;473:2300-4.

3. Kurtz S, Lau E, Watson H, Schmier J, Parvizi J. Economic burden of periprosthetic joint infection in the United States. J Arthroplast. 2012;27:61-5

4. Klouche S, Sariali E, Mamoudy P. Total hip arthroplasty revision due to infection: a cost analysis approach. Orthop Traumatol Surg Res. 2010;96:167-75.

5. Leonard HAC, Liddle AD, Burke O, Murray DW, Pandit H. Single- or two-stage revision for infected total hip arthroplasty? A systematic review of the literature. Clin Orthop Relat Res. 2014;472:1036-42.

6. Wolf M, Clar H, Friesenbichler J, Schwantzer G, Bernhardt G, Gruber G, et al. Prosthetic joint infection following total hip replacement: results of one-stage versus two-stage exchange. Int Orthop. 2014;38:1363-8.

7. Osmon D, Berbari E, Berendt A, Lew D, Zimmerli W, Steckelberg $\mathrm{J}$, et al. Diagnosis and management of prosthetic joint infection: 
clinical practice guidelines by the Infectious Diseases Society of America. Clin. Infect. Dis. 2013;56:e1-25.

8. McPherson EJ, Woodson C, Holtom P, Roidis N, Shufelt C, Patzakis M. Periprosthetic total hip infection: outcomes using a staging system. Clin. Orthop. Relat. Res. 2002;8-15.

9. Giulieri SG, Graber P, Ochsner PE, Zimmerli W. Management of infection associated with total hip arthroplasty according to a treatment algorithm. Infection. 2004;32:222-8.

10. Parvizi J, Adeli B, Zmistowski B, Restrepo C, Greenwald AS. Management of periprosthetic joint infection: the current knowledge: AAOS exhibit selection. J Bone Jt Surg Am. 2012;94:104.

11. Fink B, Vogt S, Reinsch M, Büchner H. Sufficient release of antibiotic by a spacer 6 weeks after implantation in two-stage revision of infected hip prostheses. Clin Orthop Relat Res. 2011;469:3141-7.

12. Cabo J, Euba G, Saborido A, González-Panisello M, Domínguez MA, Agulló JL, et al. Clinical outcome and microbiological findings using antibiotic-loaded spacers in two-stage revision of prosthetic joint infections. J Infect. 2011;63:23-31.

13. Classen $\mathrm{T}$, von Knoch M, Wernsmann M, Landgraeber S, Löer F, Jäger M. Functional interest of an articulating spacer in two-stage infected total knee arthroplasty revision. Orthop Traumatol Surg Res. 2014;100:409-12.

14. Corona PS, Espinal L, Rodríguez-Pardo D, Pigrau C, Larrosa N, Flores X. Antibiotic susceptibility in gram-positive chronic joint arthroplasty infections: increased aminoglycoside resistance rate in patients with prior aminoglycoside-impregnated cement spacer use. J Arthroplast. 2014;29:1617-21.

15. Gomez MM, Tan TL, Manrique J, Deirmengian GK, Parvizi J. The fate of spacers in the treatment of periprosthetic joint infection. J Bone Jt Surg Am. 2015;97:1495-502 (The American Orthopedic Association).

16. Parvizi J, Azzam K, Ghanem E, Austin MS, Rothman RH. Periprosthetic infection due to resistant staphylococci: serious problems on the horizon. Clin Orthop Relat Res. 2009;467:1732-9.

17. Schmolders J, Hischebeth GTR, Friedrich MJ, Randau TM, Wimmer MD, Kohlhof H, et al. Evidence of MRSE on a gentamicin and vancomycin impregnated polymethyl-methacrylate (PMMA) bone cement spacer after two-stage exchange arthroplasty due to periprosthetic joint infection of the knee. BMC Infect Dis. 2014;14:144.

18. Mariconda M, Ascione T, Balato G, Rotondo R, Smeraglia F, Costa GG, et al. Sonication of antibiotic-loaded cement spacers in a two-stage revision protocol for infected joint arthroplasty. BMC Musculoskelet Disord. 2013;14:193.

19. Trampuz A, Zimmerli W. Prosthetic joint infections: update in diagnosis and treatment. Swiss Med Wkly. 2005;135:243-51.

20. Esteban J, Gadea I, Pérez-Jorge C, Sandoval E, García-Cañete J, Fernandez-Roblas R, et al. Diagnosis of spacer-associated infection using quantitative cultures from sonicated antibiotics-loaded spacers: implications for the clinical outcome. Eur J Clin Microbiol Infect Dis. 2016;35:207-13.

21. Sorli L, Puig L, Torres-Claramunt R, Gonzalez A, Alier A, Knobel $\mathrm{H}$, et al. The relationship between microbiology results in the second of a two-stage exchange procedure using cement spacers and the outcome after revision total joint replacement for infection: The use of sonication to aid bacteriological analysis. Bone Jt J. 2012;94B:249-53.

22. Zimmerli W, Trampuz A, Ochsner PE. Prosthetic-joint infections. N Engl J Med. 2004;351:1645-54.

23. Marmor S, Kerroumi Y. Patient-specific risk factors for infection in arthroplasty procedure. Orthop. Traumatol. Surg. Res. 2016;102:S113-9.

24. Zmistowski B, Karam JA, Durinka JB, Casper DS, Parvizi J. Periprosthetic joint infection increases the risk of one-year mortality. J. Bone Jt Surg Am. 2013;95:2177-84.

25. Shahi A, Tan TL, Chen AF, Maltenfort MG, Parvizi J. In-Hospital mortality in patients with periprosthetic joint Infection. J. Arthroplasty. 2017;32:948-52.e1.

26. Bertazzoni Minelli E, Benini A, Samaila E, Bondi M, Magnan B. Antimicrobial activity of gentamicin and vancomycin combination in joint fluids after antibiotic-loaded cement spacer implantation in two-stage revision surgery. J Chemother. 2015;27:17-24.

27. Neumann DRP, Hofstaedter T, List C, Dorn U. Two-stage cementless revision of late total hip arthroplasty infection using a premanufactured spacer. J Arthroplast. 2012;27:1397-401.

28. Younger AS, Duncan CP, Masri BA. Treatment of infection associated with segmental bone loss in the proximal part of the femur in two stages with use of an antibiotic-loaded interval prosthesis. J Bone Jt Surg Am. 1998;80:60-9.

29. Anagnostakos K, Fürst O, Kelm J. Antibiotic-impregnated PMMA hip spacers: current status. Acta Orthop. 2006;77:628-37.

30. Sendi P, Rohrbach M, Graber P, Frei R, Ochsner PE, Zimmerli W. Staphylococcus aureus small colony variants in prosthetic joint infection. Clin Infect Dis. 2006;43:961-7.

31. Anagnostakos K, Jung J, Schmid NV, Schmitt E, Kelm J. Mechanical complications and reconstruction strategies at the site of hip spacer implantation. Int J Med Sci. 2009;6:274-9.

32. Lange J, Troelsen A, Thomsen RW, Søballe K. Chronic infections in hip arthroplasties: comparing risk of reinfection following one-stage and two-stage revision: a systematic review and metaanalysis. Clin Epidemiol. 2012;4:57-73.

33. Chen S-Y, Hu C-C, Chen C-C, Chang Y-H, Hsieh P-H. Two-stage revision arthroplasty for periprosthetic hip infection: mean Follow-Up of ten years. Biomed Res Int. 2015;2015:345475.

34. Anagnostakos K, Hitzler P, Pape D, Kohn D, Kelm J. Persistence of bacterial growth on antibiotic-loaded beads: is it actually a problem? Acta Orthop. 2008;79:302-7.

35. Chambers B, Fankhauser RA, Howard M. Bacterial growth on articulating spacers: an in vitro study. Orthopedics. 2008;31:221.

36. Chiang E-R, Su Y-P, Chen T-H, Chiu F-Y, Chen W-M. Comparison of articulating and static spacers regarding infection with resistant organisms in total knee arthroplasty. Acta Orthop. 2011;82:460-4 (Taylor \& Francis).

37. Choi H-R, Freiberg AA, Malchau H, Rubash HE, Kwon Y-M. The fate of unplanned retention of prosthetic articulating spacers for infected total hip and total knee arthroplasty. J Arthroplast. 2014;29:690-3. 